\title{
COVERT ORIENTING OF VISUOSPATIAL ATTENTION IN THE EARLY STAGES OF AGING
}

\author{
Authors: Laura Lorenzo-López, Sonia Doallo, Carmen Vizoso, Elena Amenedo, \\ Socorro Rodríguez Holguín, Fernando Cadaveira
}

This is the peer reviewed version of the following article: Lorenzo-López, L., Doallo, S., Vizoso, C., Amenedo, E., Rodríguez Holguín, S. \& Cadaveira, F. (2002). Covert orienting of visuospatial attention in the early stages of aging. Neuroreport,13(11), 1459-1462. doi: 10.1097/00001756-200208070-00022

This article may be used for non-commercial purposes in accordance with Lippincott, Williams \& Wilkins terms and conditions for use of self-archived versions. 


\title{
Covert orienting of visuospatial attention in the early stages of aging
}

\author{
Laura Lorenzo-López1', Sonia Doallo, Carmen Vizoso, Elena Amenedo, Socorro \\ Rodríguez Holguín and Fernando Cadaveira \\ Department of Clinical Psychology and Psychobiology, University of Santiago de \\ Compostela, Campus Sur S/N, 15782, Santiago de Compostela, Spain
}

\begin{abstract}
Electrophysiological and behavioral responses were recorded in healthy young (19-23 years) and older (56-66 years) subjects dur- ing the execution ofa visuospatial attention task. The objective was to test whether covert orienting of visuospatial attention (COVAT) is sensitive to the early stages of aging. All subjects responded faster to targets following valid than invalid cues. The amplitude of the P1component of visual event-related potentials (ERP) was larger to targets following central valid cues at all SOAs. Subtle age-related changes were observed in P1 amplitude under peripheral cueing. Furthermore, older subjects presented longer reaction times (RTs) and lower P1 amplitudes regardless ofthe attention condition.
\end{abstract}

Key words: Aging; Central cueing; COVAT; Event-related potentials; P1; Peripheral cueing

\section{Introduction}

Subjects are able to covertly shift their attention to specific locations in visual space in the absence of eye or head movements [1,2]. Covert orienting of visuospatial attention (COVAT) has been investigated in both behavioral and electrophysiological experiments using trial-by-trial cueing paradigms where a valid cue informs subjects about the correct position of the subsequent target stimulus, and an invalid cue directs attention to an incorrect location (the Posner task). In tasks of this kind, faster and more accurate RTs to targets following valid than invalid cues are attributed to a shift in visuospatial focus of attention to the cued location, thereby facilitating sensory processing of the target [3]. The type of spatial cue influences the nature of the attention shift. Peripheral cues appear to attract attention automatically, and are most effective at shorter stimulus-onsetasynchronies (SOAs) [4,5]. Central symbolic cues, however, appear to initiate a voluntary mode of attention shifting, and are most effective at longer SOAs [5-7].

In contrast to most behavioral experiments, few ERP studies have investigated the effects of visuospatial orienting induced by trial-by-trial cueing, with most of them

\footnotetext{
${ }^{1}$ Corresponding author

Received 1 May 2002; accepted 3 June 2002
} 
using central symbolic cueing and longer SOAs. A common finding has been the enhancement of $\mathrm{P} 1$ amplitude at occipital scalp sites to attended targets following valid cues, which has been interpreted as a sensory gain mechanism that enhances the perceptual processing of attended stimuli relative to unattended stimuli [8]. The question of whether $\mathrm{P} 1$ enhancements are also observed with peripheral valid cues remains controversial [9].

It has been speculated that spatial selective attention may be particularly sensitive to aging [10]. Behavioral visual cueing paradigms have been used to investigate selective attentional abilities in healthy older subjects, but the results of the few existing studies have been mixed and inconsistent. Some studies have reported agerelated declines in visual attention, most of them showing deficits in aspects of controlled orienting using central symbolic cues $[11,12]$. However, automatic orienting in response to peripheral cues has been found to be relatively well preserved in older adults [11-13]. In general, these studies suggest that age-related decrements in attention shifts are limited to tasks which require voluntary processing. On the other hand, no age-related differences have been also reported [14].

ERP studies can be useful in detecting age-related deficits in attentional processes, as they can help in localizing the specific mechanisms that are involved in the selection of information and which may become altered with aging. The analysis of the early sensory gain mechanism indexed by $\mathrm{P} 1$ amplitude may therefore add valuable information about the covert orienting of visual attention. So far, only one study has analyzed this question [15], although it only used central cueing conditions and long SOAs (795 ms). Young and older adults responded more quickly following valid rather than invalid cues. P1 amplitude was similarly affected by cueing in each age group.

In the present study, we measured RT and P1 amplitude under automatic and voluntary attention shift conditions (central vs. peripheral cueing) during a Posner's spatial cueing paradigm [9], to further explore the effects of aging on covert visuospatial orienting. Moreover, although it has been found that the nature of visuospatial attention shifting differs depending on the timing between the cue and the target stimulus, no ERP studies exist which have systematically examined the temporal course of covert orienting with both central and peripheral cueing paradigms. In this report, we have controlled this variable by using similar stimulus parameters and the same range of SOAs $(100,300$, 500 and $700 \mathrm{~ms}$ ) for both central and peripheral cue conditions, to assess the electrophysiological correlates of time course of exogenous and endogenous attention shifts, and the possible effects of aging on it.

\section{Materials and Methods}

Subjects: Ten young (seven females, age 20 \pm 1 years, range 19-23) and 10 older subjects (five females, age $60 \pm 3$ years, range 56-66) were tested. All were healthy, 
functioning individuals without a history of neurological or psychiatric disease, or visual impairment.

Stimuli and procedure: A fixation cross was presented continuously at the center of a computer screen. In the central cue condition, subjects were presented with a centrally located arrow cue that directed their attention toward either the left or right visual field (0.50 probability). The arrow pointed towards the upcoming target location in valid trials $(p=0.75)$ and in the opposite direction in invalid trials $(p=0.25)$. Target stimuli consisted of either long $\left(2.1 \times 0.7^{\circ}\right)$ or short $\left(1.7 \times 0.7^{\circ}\right)$ vertical bars flashed in the left or right visual field $6.4^{\circ}$ of visual angle lateral to fixation. The target locations were each defined by four continuously present dots that formed the corners of a vertical rectangle $\left(1.5 \times 1.1^{\circ}\right)$ centered on the target's position. The cueing arrow and bar stimuli were flashed for durations of 34 and $75 \mathrm{~ms}$, respectively. The interval between cue onset and target onset (SOA) varied between 100, 300, 500 and $700 \mathrm{~ms}$, and trials occurred at a rate one every $1.8 \mathrm{~s}$. In the peripheral condition, stimuli and procedures were similar to those described for the central cue condition except as specified in the following. Trials began with a peripheral cue that consisted of a brief displacement of the dots that marked one of the target locations. The four dots were removed and replaced for $50 \mathrm{~ms}$ by four dots that formed a new outline rectangle measuring $0.5 \times 1.1^{\circ}$. The original dots were then restored, giving the appearance that the continuously present marker dots had jumped toward and then away from each other in the vertical dimension. This peripheral cue occurred in the same visual field as the subsequent target bar $75 \%$ of the time (valid trials) and in the opposite visual field $25 \%$ of the time (invalid trials). The cue-target SOAs were identical to those in the central cue condition. A total of 960 trials per condition were run.

Several training trials were run at the beginning of the experimental session to ensure a good level of performance. During the recording, subjects were required to maintain central fixation and to make a discrimination of the height of the target bars, pressing a button with their left hand in response to short bars, and a button with their right hand in response to long bars, as rapidly and accurately as possible. Assignment of response keys and the order of conditions (central/peripheral cueing, SOA length) were counterbalanced across subjects. Subjects were informed of the probabilities of the valid and invalid trial types and were told to use this information to maximize their performance.

The electroencephalogram (EEG) was recorded with a NeuroScan system using scalp electrocaps (ECI, Inc.) with electrodes placed at FP1, FPz, FP2, F3, Fz, F4, F7, F8, C3, Cz, C4, CP3, CP4, P3, Pz, P4, T3, T4, T5, T6, PO3, POz, PO4, Oz, O1, O2 (10/20 International System). Four extra electrodes were fixed to the scalp, located halfway between Pz and each ear canal (PL, PR), and between $\mathrm{O} 1$ and T5 (OL), and $\mathrm{O} 2$ and T6 (OR) [16]. The active electrodes were referred to the nose and grounded with an electrode placed at nasion. Vertical and horizontal EOG activities were recorded bipolarly 
from above and below the left eye and from the outer canthi of both eyes. The EEG signals were continuously amplified (10K) and digitized at a rate of $500 \mathrm{~Hz} /$ channel, and filtered on-line with a bandpass of $0.05-100 \mathrm{~Hz}$.

For each electrode, and once ocular artifacts had been corrected, EEG epochs consisting of $900 \mathrm{~ms}$ post-stimulus and $100 \mathrm{~ms}$ pre-stimulus were obtained off-line and averaged for the target stimuli in each condition separately. Trials exceeding $7100 \mu \mathrm{V}$ were automatically excluded from the averages, as well as trials associated with incorrect responses. In order to ensure that subjects maintained their gaze at central fixation, trials containing horizontal EOG activity were also excluded from the average.

Behavioral responses were also automatically recorded on-line for all subjects in all of the experimental conditions. Only RT values associated with correct responses were considered for data analyses.

Data analysis: For conventional statistical analyses, peak amplitudes of $\mathrm{P} 1$ were automatically measured relative to the $100 \mathrm{~ms}$ baseline at PL, PR, OL, OR, T5, and T6 electrodes using a latency window of $75-165 \mathrm{~ms}$. This latency window was adapted considering the interval between which $\mathrm{P} 1$ appeared in the corresponding grand mean waveforms of each age group.

P1 and RT data were subjected to mixed model ANOVAs in which age (young, older) was entered as the between-subjects factor. The within-subject factors were bar height (long, short), visual field (left, right), validity (valid, invalid), cue type (central, peripheral), and SOA $(100,300,500,700 \mathrm{~ms})$. For $\mathrm{P} 1$ analyses the additional factor of electrode (PL, PR, T5, T6, OL, OR) was entered. Whenever appropriate, degrees of freedom were corrected by the conservative Greenhouse-Geisser estimate.

In order to test the possibility of age-related and/or attention-related changes in the current source distribution of P1 amplitude, a common average reference was calculated by averaging the data, time point by time point, for all 30 active electrodes in each subject and condition. Maps were computed using brain electromagnetic source analysis (BESA22) [17]. Scalp potentials rereferenced to the common average, excluding the EOG electrodes, were interpolated for mapping using the surface spline method. The current source density (CSD) maps were computed with the spherical spline interpolated data at a single time point where P1 was largest in the grand mean waveforms of each age group and condition.

\section{Results}

Behavioral data: There was a significant main effect of age $(F(1,18)=18.13, p=0.0001)$ on correct responses, with slower reaction times in the older group (young $563 \pm 55 \mathrm{~ms}$; older $697 \pm 83 \mathrm{~ms}$ ). The effect of cue validity was significant for both young and older 
subjects $(F(1,18)=60.96, p=0.0001)$, reflecting faster reaction times for valid cues (young valid $547 \pm 56 \mathrm{~ms}$; older valid $670 \pm 77 \mathrm{~ms}$ ) and slower reaction times for invalid cues (young invalid $579 \pm 55 \mathrm{~ms}$; older invalid $724 \pm 91 \mathrm{~ms}$ ) in all experimental conditions.

$P 1$ data: There were no significant effects of age on $P 1$ amplitude $(F(1,18)=2.06, p=$ $0.17)$. There was a significant effect of electrode $(F(5,90)=10.56, p<0.0001, \varepsilon=0.61)$, reflecting $\mathrm{P} 1$ maximum amplitudes at occipital electrodes $\mathrm{OL}$ and $\mathrm{OR}$. $\mathrm{P} 1$ amplitude was generally higher with peripheral cueing $(F(1,18)=6.73, p=0.02)$, and was maximum at $300 \mathrm{~ms} \mathrm{SOA}$, and minimum at $500 \mathrm{~ms} \operatorname{SOA}(\mathrm{F}(3,54)=14.51, \mathrm{p}<0.0001, \varepsilon=0.62)$. Finally, a significant cue type $x$ validity interaction was observed $(F(1,18)=4.61, p=$ 0.04 ), indicating that enhancements of $P 1$ amplitude with valid targets were only observed in the central cue condition (Fig. 1).

Scalp distribution analyses showed similar distributions of $\mathrm{P} 1$ current sources in the two age groups with central cueing conditions. With peripheral cueing, young subjects showed more density in P1 current sources to valid cues under $100 \mathrm{~ms}$ SOA, and no differences with longer SOAs. However, the maps of older subjects indicated a general trend towards more density in $\mathrm{P} 1$ sources to invalid cues for all SOAs. Moreover, a general reduction of current source density at the latency of P1 was observed in older subjects compared to younger subjects across all conditions, indicating global amplitude reductions regardless of the attention condition (Fig. 2).

\section{Discussion}

As in previous studies, our investigation showed that older subjects responded more slowly than young subjects $[12,14,15,18]$. It has been argued that this may be explained by a selective slowing of response-related processes in elderly subjects, rather than by specific deficits in attention [10]. As has been demonstrated in previous studies, both older and younger subjects were faster at discriminating between targets following valid than invalid cues. This suggests that the efficiency of cue-based shifts of visuospatial attention is relatively resistant to the effects of aging, at least in its early stages, and that response execution is faster when attention has been previously shifted to a valid location.

Regarding electrophysiological data, the only previous study which analyzed P1 amplitude changes with age in COVAT failed to observe any changes in this parameter [15]. However, the topographic analyses in the present report shows both attentionrelated and attention-independent changes with age. The attention-related changes reflected different trends in the temporal course of sensory gain mechanisms depending on cueing type. Young subjects presented automatic attentional shifts to peripheral cues only with the shortest SOA, whereas the older group displayed an overall trend towards automatic shifts to invalid peripheral cues independently of the timing between cue and 
target stimuli. Besides this, overall P1 amplitude reductions were observed in older subjects, regardless of validity, cue type, or timing between the cue and the target. Similar findings have been previously observed with auditory ERPs [19], indicating the existence of both attention-related and attention-independent general changes with age which may underlie the basic functioning of visual sensory processing under both attention and unattention conditions.

Enhancement of P1 amplitudes in valid trials with central cueing has previously been reported in different studies $[9,16,20]$ that interpreted this effect as a sign of facilitated visual sensory processing. The question of whether validity effects are also observed in $\mathrm{P} 1$ amplitude with peripheral cueing is still controversial. The present results shed light on this matter, as they show that with peripheral cueing, and in young subjects, there is a trend to enhance visual sensory processing in valid trials only at short SOAs, which agrees with the postulated automatic nature of attention shifts in these cueing conditions.

\section{Conclusion}

Our study shows that increasing age is associated with a generalized slowing of behavioral responses under visuospatial attention conditions. The results on sensory gain mechanisms indicate the coexistence of age-related overall reductions in the magnitude of sensory processing and temporal course changes in automatic shifts of visuospatial attention.

\section{References}

1. Posner MI. Q J Exp Psychol 32, 3-25 (1980).

2. Posner MI, Nissen MJ and Ogden WC. Attended and unattended processing modes: the role of set for spatial location. In: Pick HL and Saltzman EJ, eds. Modes of Perceiving and Processing Information. Hillsdale, NJ: Lawrence Erlbaum; 1978, pp. 137-157.

3. Hawkins HL, Hillyard SA, Luck SJ et al. J Exp Psychol: Hum Percept Perform 16, 802811 (1990).

4. Danckert J and Maruff P. Percept Psychophys 59, 500-508 (1997).

5. Yantis S and Jonides J. J Exp Psychol: Hum Percept Perform 16, 121-134 (1990).

6 . Jonides J. Voluntary versus automatic control over the mind's eye movement. In: Long $\mathrm{JB}$ and Baddeley $\mathrm{AD}$, eds. Attention and performance IX. Hillsdale, $\mathrm{NJ}$ : Lawrence Erlbaum; 1981, pp. 187-203.

7. Müller HJ and Rabbitt PMA. J Exp Psychol: Hum Percept Perform 15, 315-330 (1989).

8. Mangun GR, Hansen JC and Hillyard SA. The spatial orienting of attention: sensory facilitation or response bias? In: Johnson R, Rohrbaugh JR and Parasuraman R, eds. Current Trends in Event-related Potential Research (EEG Suppl. 40). New York: Elsevier Science; 1987, pp. 118-124. 
9. Hillyard SA, Luck SJ and Mangun GR. The cuing of attention to visual field locations: Analysis with ERP recordings. In: Heinze HJ, Münte TF and Mangun GR, eds. Cognitive Electrophysiology. Boston, MA: Birkhaüser; 1994, pp. 1-25.

10. Hartley AA. Attention. In: Craik FIM and Salthouse TA, eds. The Handbook of Aging and Cognition. Hillsdale, NJ: Lawrence Erlbaum; 1992, pp. 3-49.

11. Greenwood PM, Parasuraman R and Haxby JV. Neuropsychologia 31, 471-485 (1993).

12. Hartley AA, Kieley JM and Slabach EH. J Exp Psychol Hum Percept Perform 16, 523-538 (1990).

13. Robinson DL and Kertzman C. Neuropsychologia 28, 291-301 (1990).

14. Folk CL and Hoyer WJ. Psychol Aging 7, 453-465 (1992).

15. Curran T, Hills A, Patterson MB et al. Neuropsychologia 39, 288-301 (2001).

16. Mangun GR and Hillyard SA. J Exp Psychol: Hum Percept Perform 17, 1057-1074 (1991).

17. Scherg $M$ and Berg P. Brain Electromagnetic Source Analysis. Munich: MEGIS; 1996.

18. Yamaguchi S, Tsuchiya H and Kobayashi S. Cogn Brain Res 3, 41-49 (1995).

19. Amenedo E and Díaz F. Neuroreport 10, 2383-2388 (1999).

20. Anllo-Vento L. Int J Neurosci 80, 353-370 (1995).

Acknowledgements: This study was supported by the Spanish Ministerio de Ciencia yTecnolog|ía (MCYT-DGI) grant BSO2000-0041; and by Xunta de Galicia grants PGIDT01PXI21101PN and PGIDT00PXI21102PR. We would like to thank Neurobehavioral Systems, Inc. for their support with their Presentation software. 
Figures
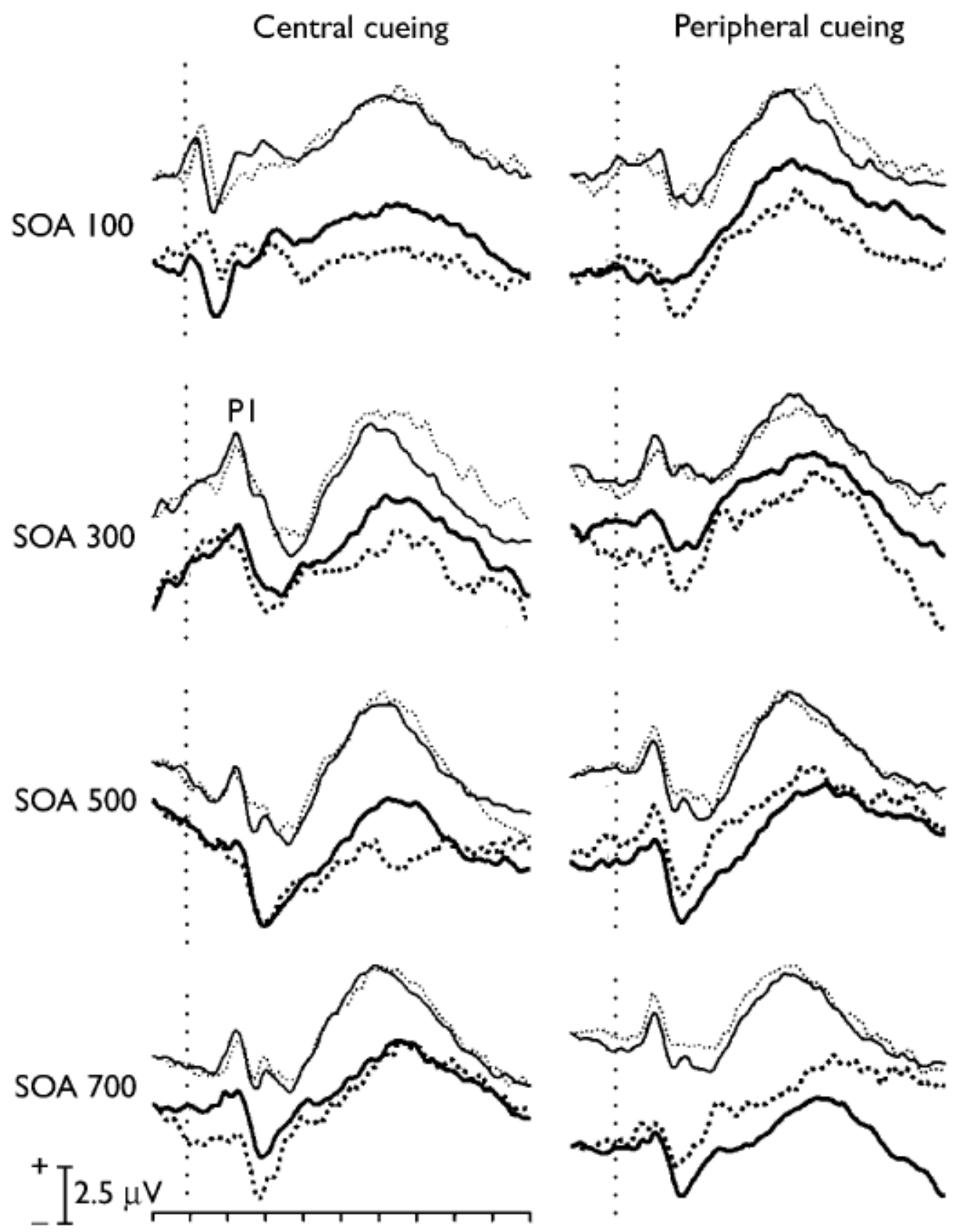

$-1000100200300400500600700800900 \mathrm{~ms}$

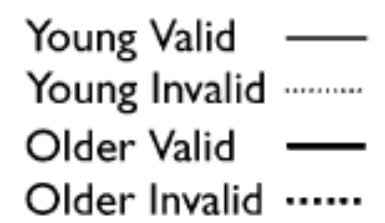

Figure 1. Grand-mean ERPs for each age group showing P1 elicited to valid and invalid stimuli at the occipital electrode contralateral to the stimulation field (OR). 

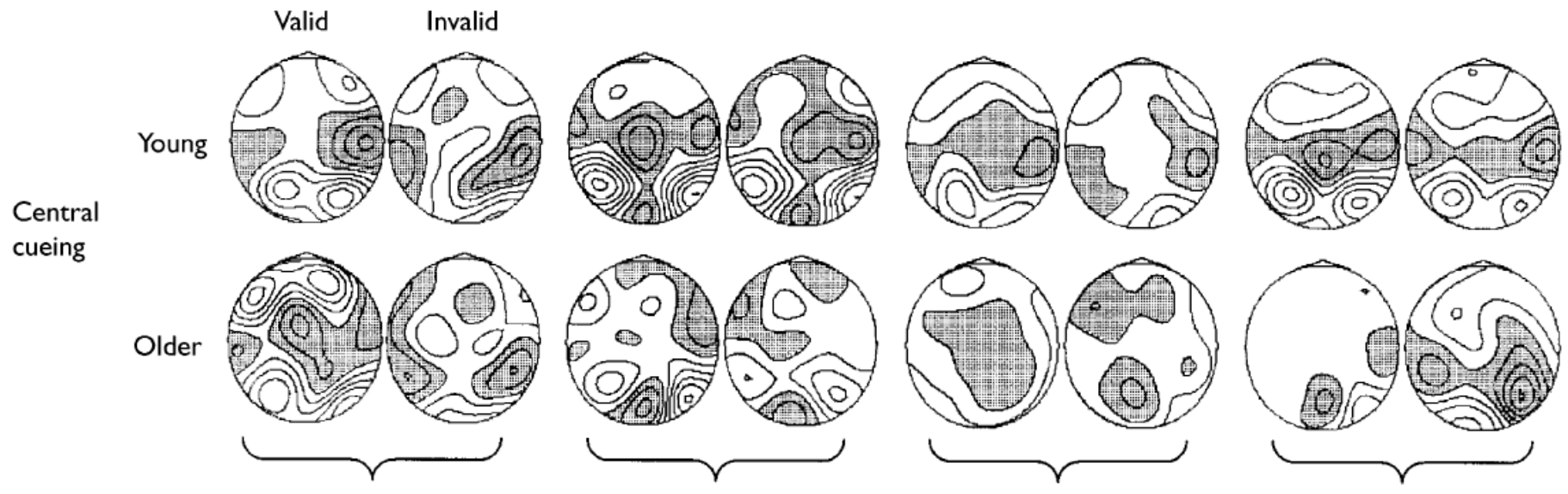

SOA

100
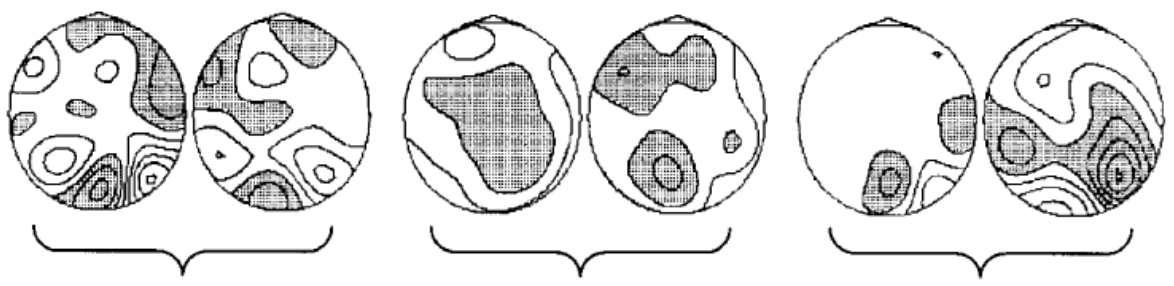

300

500

$700 \mathrm{~ms}$
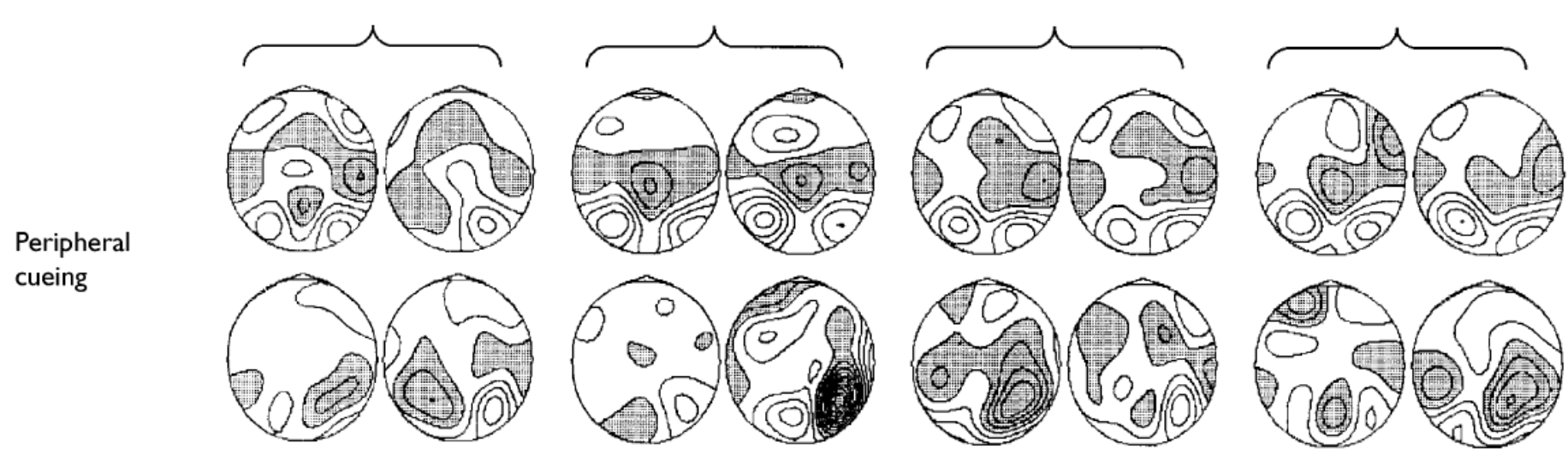

Figure 2. CSD maps for $P 1$ in the two age groups across attention condition. Isopotential lines are separated by $0.05 \mu \mathrm{V} / \mathrm{cm}^{2}$. Shaded areas indicate current sinks, and unshaded areas indicate current sources. Maps represent activity to stimulation given on the left visual hemifield. 